\title{
A portrait of yeast
}

The importance of yeast for fermentation has shaped our shared history with this organism for thousands of years. By analysing the genomes of dozens of isolates of domestic and wild yeast two studies now document the effect of evolutionary processes on the yeast genome - the result is not only a detailed polymorphism map for this medically and commercially important group of organisms, but also evidence of the influence of humans on the genome structure of domesticated yeast.

To investigate the poorly understood relationship between phenotypic adaptation and genome variation, Gianni Liti and colleagues sequenced the genomes of Saccharomyces cerevisiae from worldwide locations and compared

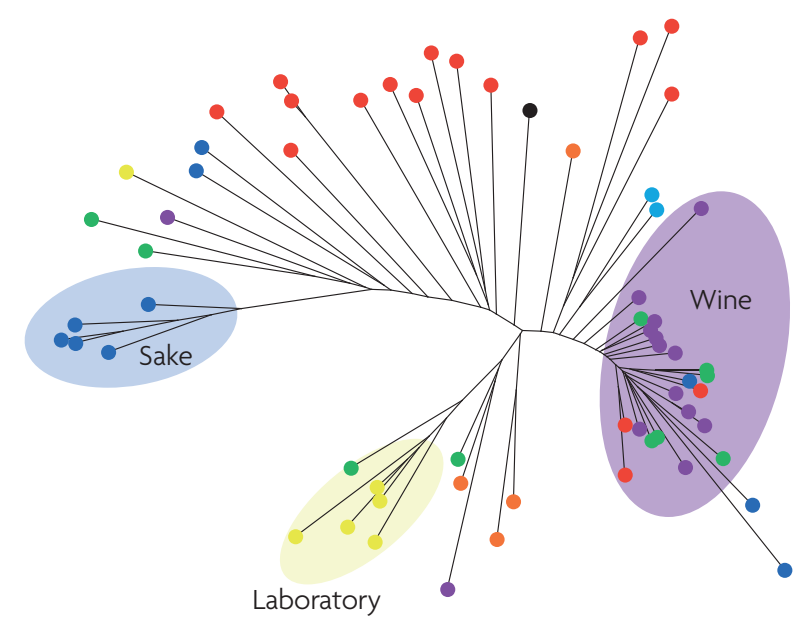

Neighbour joining tree of 63 Saccharomyces cerevisiae strains. Image is modified, with permission, from Schacherer, J. et al. Nature (doi:10.1038/nature07670) (c) (2009) Macmillan Publishers Ltd. All rights reserved. them to those of the closely related but non-domesticated wild species Saccharomyces paradoxus.

This genome-wide survey provides a detailed picture of variation in gene content between $>70$ strains, as well as genomic variants such as new ORFs and copy number and insertiondeletion polymorphisms. The 35 S. paradoxus isolates fall into three phylogenetically distinct populations, as inferred from SNP-based neighbour joining trees; by contrast, only 20 of the 36 sampled S. cerevisiae strains fall into five 'pure' lineages - the others have mosaic genomes, perhaps a result of lineage mixing caused by human activity. In addition, most strains of $S$. cerevisiae have a higher phenotypic variance than $S$. paradoxus strains, and so probably they occupy a larger ecological niche. Although these data can be used to infer a more detailed phylogenetic history of yeast strains, they also serve as a resource for fine mapping interesting phenotypic differences (the Saccharomyces Genome Resequencing Project).

Another example of the contribution of large-scale genotyping technologies to evolutionary functional genomics is provided by a second paper; here, the authors define the polymorphism structure of 63 S. cerevisiae strains by using wholegenome tiling microarrays. Over 100,000 segregating SNPs were used to determine, for the first time, several population genetic parameters, including linkage disequilibrium structure and patterns of deletion polymorphisms across geographically and ecologically dispersed samples. Because segregating sites are located every 100 bp, the SNP map is dense enough to be used as a resource for genome-wide association studies (the YSB database).

The phylogenetic relationships inferred from the polymorphic sites support related conclusions by Liti et al. that wine making, sake making and laboratory strains fall into three separate clusters (see the figure), suggesting that they arose from three distinct domestication events. By contrast, clinical isolates have arisen multiple times, suggesting that many non-clinical strains can opportunistically acquire the ability to colonize human tissues.

Although much information exists on the interspecies variation between Saccharomyces and other species within the same phylum, the polymorphism maps developed by these two studies offer the first opportunity to study phenotypegenotype correlations within Saccharomyces itself.

Tanita Casci

ORIGINAL RESEARCH PAPERS Liti, G. \& Carter, D. M. et al. Population genomics of domestic and wild yeasts. Nature $11 \mathrm{Feb} 2009$ (doi:10.1038/nature07743) | Schacherer, J. \& Shapiro, J. A. et al. Comprehensive polymorphism survey elucidates population structure of Saccharomyces cerevisiae. Nature 11 Feb 2009 (doi:10.1038/nature07670).

WEBSITES

Yeast SNPs Browser (YSB) database: http://gbrowse.princeton.edu/yeastSNP Saccharomyces Genome Resequencing Project: www.sanger.ac.uk/Teams/Team118/sgrp 